\title{
Post-Retrieval Extinction Attenuates Cocaine Memories
}

\author{
Gregory C Sartor' and Gary Aston-Jones*,' \\ 'Department of Neurosciences, Medical University of South Carolina, Charleston, SC, USA
}

\begin{abstract}
Recent studies have shown that post-retrieval extinction training attenuates fear and reward-related memories in both humans and rodents. This noninvasive, behavioral approach has the potential to be used in clinical settings to treat maladaptive memories that underlie several psychiatric disorders, including drug addiction. However, few studies to date have used a post-retrieval extinction approach to attenuate addiction-related memories. In the current study, we attempted to disrupt cocaine-related memories by using the post-retrieval extinction paradigm in male Sprague Dawley rats. Results revealed that starting extinction training I h after cocaine contextual memory was retrieved significantly attenuated cocaine-primed reinstatement of conditioned place preference (CPP) and relapse of cocaine CPP (drug-free and cocaine-primed) following 30 days of abstinence. However, animals that did not retrieve the contextual cocaine memory before extinction training, or animals that began extinction training $24 \mathrm{~h}$ after retrieval (outside of the reconsolidation window), demonstrated normal cocaine CPP. Conversely, animals that received additional CPP conditioning, rather than extinction training, I h after reactivation of cocaine memory showed enhanced cocaine CPP compared with animals that did not reactivate the cocaine memory before conditioning. These results reveal that a behavioral manipulation that takes advantage of reconsolidation and extinction of drug memories may be useful in decreasing preference for, and abuse of, cocaine.

Neuropsychopharmacology (2014) 39, 1059-1065; doi: I0. I038/npp.2013.323; published online 18 December 2013
\end{abstract}

Keywords: post-retrieval extinction; conditioned place preference; cocaine; relapse

\section{INTRODUCTION}

Drug addiction is considered by many as a disorder of aberrant learning and memory (Hyman, 2005; Torregrossa et al, 2011). With each drug use, environmental cues and contexts become strongly associated with the rewarding effects of the drug. Over time the cues/contexts alone are capable of stimulating drug-related memories that induce craving and relapse (Shaham et al, 2003; Volkow et al, 2006). Thus, targeting drug-associated memories has been a major focus in addiction research, as therapies capable of disrupting such memories carry important implications for the treatment of addiction.

Consolidated memories were once thought to be permanently encoded memory traces (Glickman, 1961; McGaugh, 1966), but more recent studies have shown that some memories (including memories associated with addiction) can be rendered labile following retrieval/reactivation (Nader et al, 2000; Lee et al, 2005). This labile property, termed reconsolidation, is a time-dependent process $(<6 \mathrm{~h})$, in which consolidated memories become transiently unstable shortly after retrieval and are susceptible to

\footnotetext{
*Correspondence: Dr G Aston-Jones, Department of Neurosciences, Medical University of South Carolina, 173 Ashley Avenue, MSC 510 , Charleston, SC 29425, USA, Tel: + | 843792 1800, Fax: + I 843 792 4423, E-mail: astong@musc.edu

Received 5 June 2013; revised 15 November 2013; accepted 18 November 2013; accepted article preview online 21 November 2013
}

updating or disruption. In recent years, several studies have exploited this reconsolidation process by injecting animals with amnestic agents either before or immediately after retrieval (during reconsolidation) to attenuate previously consolidated drug-related memories (Lee et al, 2005; Lee et al, 2006; Valjent et al, 2006; Fricks-Gleason and Marshall, 2008; Milton et al, 2008; Fuchs et al, 2009; Sanchez et al, 2010). However, many of these amnestic agents (eg, protein synthesis inhibitors) are not suitable for human use, and thus other reconsolidation-based behavioral or pharmaceutical avenues are needed for the treatment of addiction.

As an alternative to harsh amnestic agents, Monfils et al (2009) recently described a noninvasive, behavioral approach to attenuate memories by post-retrieval extinction training. In this procedure, fear-conditioned animals were given an isolated retrieval trial followed by extinction training at time points inside $(10$ or $60 \mathrm{~min})$ or outside $(24 \mathrm{~h})$ of the reconsolidation window. They found that presenting an extinction session during, but not outside of, the reconsolidation window attenuated reinstatement, renewal, and spontaneous recovery of fear memories (Monfils et al, 2009). Subsequent studies in other laboratories have shown that post-retrieval extinction reduces fear and reward-related memories in both animals and humans (Clem and Huganir, 2010; Schiller et al, 2010; Flavell et al, 2011; Rao-Ruiz et al, 2011; Xue et al, 2012), indicating that these methods have the potential to be translated into clinical applications. However, other studies have failed to 
replicate such findings in fear-related memories (Chan et al, 2010; Costanzi et al, 2011; Ishii et al, 2012). Additionally, reports have challenged the reconsolidation theory behind post-retrieval extinction (Millan et al, 2013) and the notion that memories are completely erased following postretrieval extinction ( $\mathrm{Ma}$ et al, 2012). In the current study, we tested whether single-session post-retrieval extinction or post-retrieval reconditioning is able to alter cocaine-related memories in rats using the conditioned place preference (CPP) paradigm.

\section{MATERIALS AND METHODS}

\begin{abstract}
Animals
Male Sprague Dawley rats (initial weight $\sim 300-325 \mathrm{~g}$, Charles River Laboratories, Raleigh, NC) were pair-housed under a reversed $12 \mathrm{~h} / 12 \mathrm{~h}$ light/dark cycle and had ad libitum access to food and water. Animals were housed in a temperature- and humidity-controlled, AAALACaccredited, animal facility at the Medical University of South Carolina (MUSC). All experiments were approved by the Institutional Animal Care and Use Committee at MUSC and conducted according to specifications of the NIH as outlined in the Guide for the Care and Use of Laboratory Animals. A total of 84 rats were used in these experiments.
\end{abstract}

\section{Conditioned Place Preference}

CPP experiments utilized methods previously employed by our laboratory (Harris and Aston-Jones, 2003b; Harris et al, 2004; Harris et al, 2005; Sartor and Aston-Jones, 2012b; Sartor and Aston-Jones, 2012a). Briefly, the CPP apparatus consisted of two distinct compartments that were separated by a removable partition. In a pre-test acclimation session, animals were allowed free access to both sides of the chamber for $15 \mathrm{~min}$ via a doorway in the partition. The time spent on each side of the chamber was recorded automatically. None of the animals had an initial bias for either side of the chamber on the pre-test as indicated by the group average. After 2 days, animals were conditioned for 1 (Experiment 3) or 3 days (Experiment 1 and 2). During conditioning, the animals were injected with cocaine $(10 \mathrm{mg} / \mathrm{kg}$, NIDA Research Triangle Park, NC) and confined to one side of the chamber by a solid partition for $30 \mathrm{~min}$, or injected with saline and confined to the other side of the chamber for $30 \mathrm{~min}$. Injections were given on both sides of the apparatus for each animal in a balanced fashion in morning and afternoon sessions (at least $4 \mathrm{~h}$ apart). Following conditioning, animals received retrieval-extinction or retrieval-reconditioning training described below. In all experiments, the time spent on both sides of the chamber was automatically measured via photobeam breaks and custom software.

In the retrieval session for all experiments, rats were placed in the cocaine- (Ret) or saline-paired (No Ret) side for $3 \mathrm{~min}$ (drug-free), and in the extinction session (also called initial CPP test) rats had free access to both sides of the chamber for 15 min (drug-free). In Experiment 1, 1 day after conditioning, animals were confined to the cocainepaired (retrieval group) or saline-paired chamber (no retrieval group) for $3 \mathrm{~min}$ and were then placed back into their home cage. After $1 \mathrm{~h}$, animals received a 15-min drugfree extinction test in which animals had free access to both sides of the chamber via a doorway in the partition. On subsequent days, animals received one such extinction test per day until preference for the cocaine-paired was extinguished $(<75 \mathrm{~s}$ spent in the cocaine-paired side for 2 consecutive days). The next day, animals received a reinstatement test in which a cocaine injection $(10 \mathrm{mg} / \mathrm{kg})$ was given immediately before the CPP test.

In Experiment 2, 1 day after conditioning, animals were confined to the cocaine-paired (retrieval group) or salinepaired chamber (no retrieval group) for $3 \mathrm{~min}$, and then placed back into their home cage. Animals then received a 15-min extinction session (also called initial CPP test) 1 or $24 \mathrm{~h}$ later. After the extinction session, animals were returned to their home cage and were tested again for relapse of cocaine CPP after 30 days of abstinence in a drugfree or cocaine-primed $(10 \mathrm{mg} / \mathrm{kg})$ state.

In Experiment 3, 1 day after receiving a single conditioning session, conditioned animals were confined to the cocaine-paired (retrieval group) or saline-paired chamber (no retrieval group) for $3 \mathrm{~min}$ and then returned to their home cage. After $1 \mathrm{~h}$, animals received a 30 -min cocaine $\mathrm{CPP}$ reconditioning session. The next day all animals were tested for cocaine preference. In these experiments, animals were only given one conditioning session before the retrieval-reconditioning manipulation to avoid a ceiling effect with preference scores.

\section{Data Analysis}

Preference scores were calculated as the time spent in the cocaine-paired side minus the time spent in the salinepaired side on the CPP test. The resulting preference scores were compared within or between groups using Student's $t$-test, one-way analysis of variance (ANOVA) followed by Newman-Keuls post-hoc test, or two-way ANOVA followed by Bonferroni post-hoc test. GraphPad Prism v5 was used for statistical analysis. The risk of Type 1 error $(\alpha)$ was set at $P<0.05$.

\section{RESULTS}

Experiment 1: The Effects of Post-Retrieval Extinction on Cocaine-Induced Reinstatement of CPP

Experiment 1 was designed to test whether post-retrieval extinction training altered reinstatement of extinguished cocaine CPP (Figure 1a). No differences in initial cocaine CPP scores were observed between Ret $(n=8)$ and No Ret $(n=7)$ groups $\left(t_{13}=0.66, P>0.05\right)$. However, rats that were placed in the cocaine-paired side on the retrieval session (Ret) did not reinstate cocaine CPP when subsequently tested with a cocaine prime, whereas rats that did not retrieve the cocaine memory before extinction (No Ret) robustly reinstated cocaine CPP $\quad\left(t_{13}=3.1, \quad P<0.01\right.$; Figure 1b). The lack of reinstatement observed in the Ret group is not likely due to an enhancement of extinction, as the rate of extinction was similar in the two groups $\left(t_{13}=0.52, P>0.05\right.$; Figure $\left.1 c\right)$. 
a

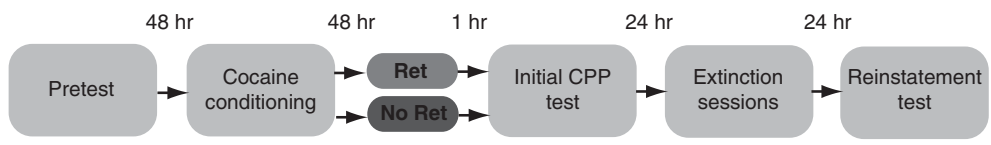

b

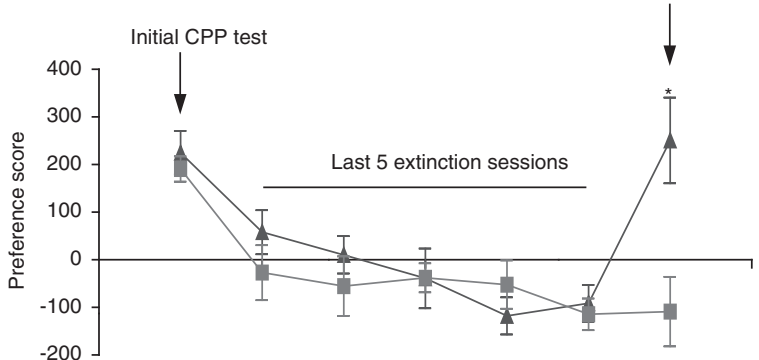

C

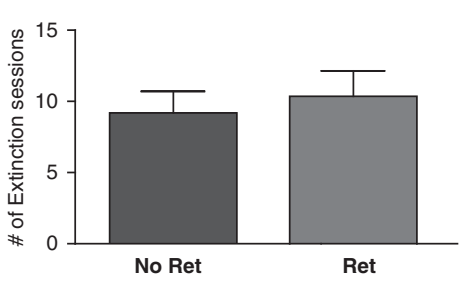

Figure I Post-retrieval extinction training blocks cocaine-primed reinstatement of CPP. (a) Schematic of the experimental procedure. Two days after cocaine conditioning, animals were exposed to the cocaine- (Ret) or saline-paired (No Ret) context for 3 min, and then received an extinction test I $\mathrm{h}$ later. On subsequent days, animals received one extinction test per day until preference for the cocaine-paired was extinguished, and then extinguished animals received a cocaine-primed reinstatement test the next day. (b) Extinction training $\mathrm{I} h$ after retrieval of a cocaine contextual memory (during the reconsolidation window, Ret group, $n=8$ ) significantly blocked cocaine-primed reinstatement of a cocaine preference (at $2 n d$ arrow) compared with animals that did not retrieve cocaine memory before extinction (No Ret, $n=7$ ) (* $P<0.0$ I indicates a significant difference from Ret group by $t$-test). No significant difference in initial expression of cocaine preference was observed between the two groups (at first arrow; $P>0.05$ ). (c) The number of sessions to reach extinction criteria did not differ between groups $(P>0.05)$. Data are expressed as means ( \pm SEM).

a
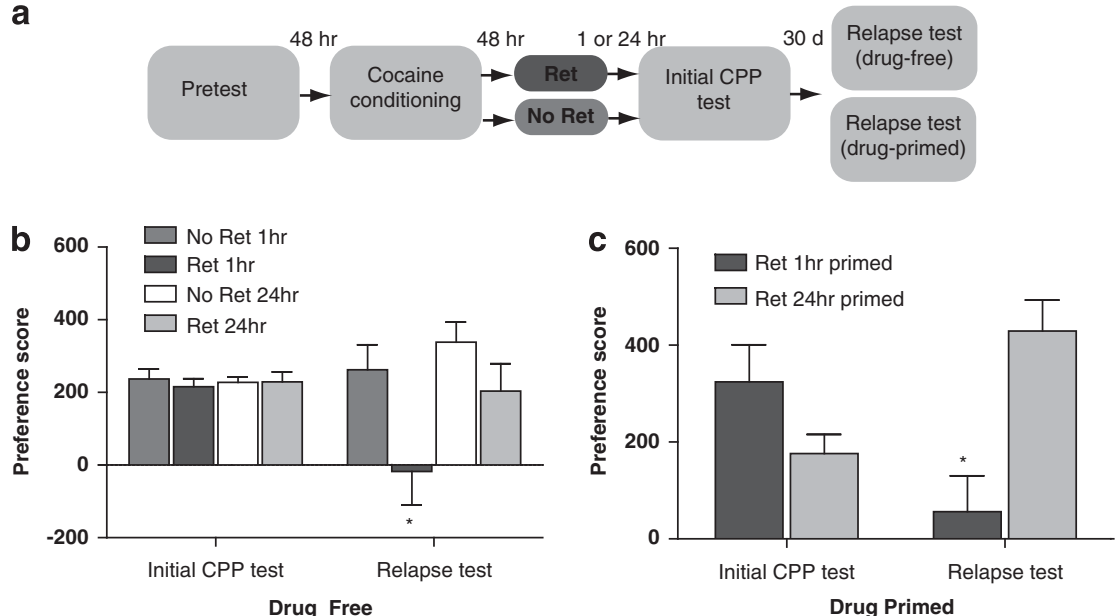

Figure 2 Post-retrieval extinction attenuates relapse of cocaine CPP following abstinence. (a) Schematic of the experimental procedure. Two days after a conditioning, animals were confined to the cocaine- (Ret) or saline-paired (No Ret) context for 3 min, and then received an extinction test I h later. Following 30 days of abstinence, animals were again tested for relapse of cocaine CPP in a drug-free or drug-primed test. (b) Presenting an extinction session I h after retrieval of cocaine contextual memory (Ret I h, $n=7$, Ret I h primed, $n=10$ ) significantly attenuated relapse of both drug-free and (c) cocaineprimed CPP following abstinence compared with animals that did not receive retrieval pairing I or $24 \mathrm{~h}$ before extinction training (No Ret I h, $n=8$; No Ret $24 \mathrm{~h}, n=5$ ), or to animals that began extinction training outside the reconsolidation window ( $24 \mathrm{~h}$ after retrieval pairing; Ret $24 \mathrm{~h}, n=9 ;$ Ret $24 \mathrm{~h}$ primed, $n=7)$. *P $<0.05$ indicates a significant difference from other relapse test scores via Bonferroni post-hoc test. Data are expressed as means $( \pm$ SEM).

\section{Experiment 2: The Effects of Post-Retrieval Extinction on Relapse of Cocaine CPP Following Abstinence}

In Experiment 2, we examined whether post-retrieval extinction altered relapse of cocaine CPP following 30 days of abstinence (Figure 2a). Initial preference scores did not differ between groups (F values $<0.6, P>0.05$ ). However, both drug-free (Figure $2 \mathrm{~b}$ ) and cocaine-primed (Figure 2c) CPP relapse scores were significantly reduced in animals that received an extinction test $1 \mathrm{~h}$ after a retrieval session (Ret
$1 \mathrm{~h}, n=7$; Ret $1 \mathrm{~h}$ primed, $n=10$ ) compared with animals that did not retrieve the cocaine memory (placed in the saline-paired side for a no retrieval session; No Ret $1 \mathrm{~h}$ and $24 \mathrm{~h}, n=8$ and 5, respectively; Figure $2 \mathrm{~b}$ and c). Similarly, animals that had one extinction test $1 \mathrm{~h}$ after a retrieval session subsequently exhibited less preference than animals that received extinction followed by retrieval outside of the reconsolidation window (Ret $24 \mathrm{~h}$ and Ret $24 \mathrm{~h}$ primed, $n=9$ and 7, respectively) (drug-free: $\mathrm{F}_{3,50}=2.8, P<0.05$, Figure $2 \mathrm{~b}$; drug primed: $F_{1,30}=13.35, P<0.01$, Figure $2 c$ ). 


\section{Experiment 3: The Effects of Post-Retrieval Reconditioning on Cocaine CPP}

Although the above and other results show that postretrieval extinction can be used to attenuate reward-related memories, it is unclear whether post-retrieval reconditioning can strengthen or enhance cocaine-related memories. In these experiments, animals were given one conditioning session, rather than three sessions, to avoid a CPP ceiling effect. The next day, animals were briefly confined to the cocaine- (Ret, $n=7$ ) or saline-paired side (No Ret, $n=7$ ). They then were reconditioned with cocaine $(10 \mathrm{mg} / \mathrm{kg}$, i.p.) $1 \mathrm{~h}$ later, and cocaine place preference was tested the next day (Figure 3a). Animals that were reconditioned following placement in the cocaine-paired side on the retrieval session (Ret) showed a significant increase in CPP scores compared with animals that were placed in the saline-paired side on the retrieval session (No Ret) before reconditioning $\left(t_{12}=3.0, P<0.05\right)$, indicating that post-retrieval reconditioning may enhance reward-related memories (Figure $3 \mathrm{~b}$ ).

\section{DISCUSSION}

\section{Overview of Results}

The current studies provide evidence that post-retrieval extinction training is an effective, noninvasive method to attenuate cocaine-related contextual memories. First, we demonstrated that commencing extinction training $1 \mathrm{~h}$ after cocaine contextual memory was retrieved significantly attenuated cocaine-primed reinstatement of CPP. Animals that did not retrieve the memory before extinction strongly reinstated cocaine $\mathrm{CPP}$ after a cocaine prime. The reduction in reinstatement does not appear to be a result of enhanced extinction, as the rate of extinction did not differ between groups. Second, we found that retrieval before a single extinction session had long-lasting effects on drug memory, as this manipulation reduced relapse of drug-free and
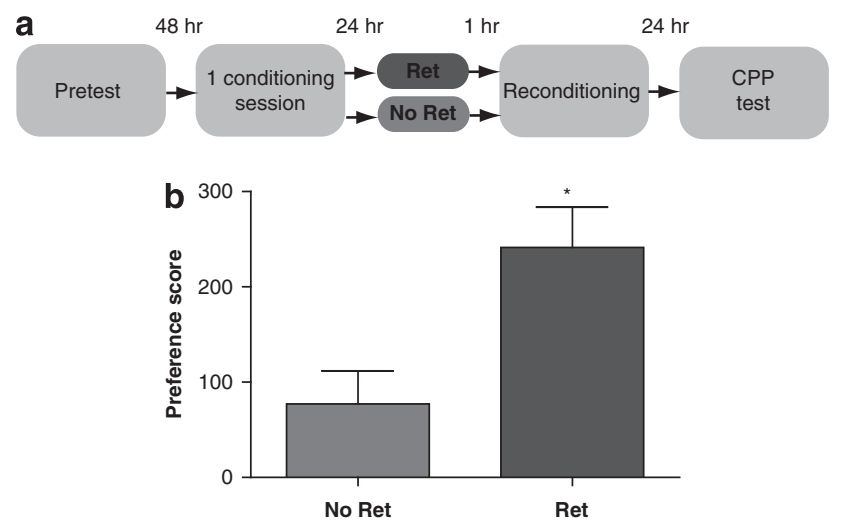

Figure 3 Cocaine conditioning during reconsolidation enhances cocaine CPP. (a) Schematic of experimental procedure. One day after a single conditioning session, animals were exposed to the cocaine- (Ret, $n=7$ ) or saline-paired context (No Ret, $n=7$ ) for 3 min, and were reconditioned I h later. Animals were tested for cocaine preference the following day. (b) A significant increase in cocaine CPP was observed when animals were reconditioned following reactivation of cocaine memory $(* P<0.05$ indicates a significant difference from No Ret group by $t$-test). Data are expressed as means $( \pm$ SEM). cocaine-primed CPP following 30 days of abstinence. This long-lasting effect is particularly important for the treatment of addiction because drug-related memories that elicit craving and relapse may persist even after prolonged abstinence (Gawin and Kleber, 1986). Third, we showed that reconditioning, rather than extinction, during reconsolidation (following retrieval of the cocaine-context memory) increased CPP expression, a finding that is consistent with Millan et al (2013) who showed the retrievalextinction procedure potentiated reacquisition of alcoholic beer self-administration. Enhancement of reward-related memories, particularly natural rewards (food, social interaction, etc) may be used as a treatment for addiction, as motivation for natural rewards have been shown to be diminished following chronic drug use (Barr et al, 1999; Harris and Aston-Jones, 2003a). Thus, by strengthening natural rewards that compete with drug memories, postretrieval reconditioning could possibly be used to reduce drug relapse. Together, these results indicate that retrievalextinction manipulations can be employed to alter cocainerelated behaviors and could potentially be utilized for the treatment of drug addiction.

\section{Post-Retrieval Extinction as a Potential Treatment for Addiction}

Cues and contexts associated with drug use are major contributing factors to craving and relapse (Shaham et al, 2003). Cue-exposure therapy (CET), an extinction-based treatment performed in a clinical setting, was developed in an attempt to diminish cue-drug associations and reduce relapse in human addicts (O'Brien et al, 1990). Unfortunately, this type of extinction therapy had limited success in preventing relapse in humans or animals (Conklin and Tiffany, 2002; Crombag and Shaham, 2002), signifying a need for alternative cognitive-behavioral strategies for the treatment of addiction. The recent development of postretrieval extinction therapy, however, may offer a more efficacious method of reducing addiction-related memories (Auber et al, 2013; Hutton-Bedbrook and McNally, 2013). In this paradigm, the strengths of both extinction and reconsolidation are combined by presenting an extinction session during the reconsolidation process, when the memory is labile, to disrupt the consolidated memory trace. Because post-retrieval extinction is thought to directly perturb drug memories, rather than forming new memories to suppress drug memories (as in extinction therapy), this new approach may be more effective at reducing relapse compared with extinction alone (Monfils et al, 2009). Indeed, using the post-retrieval extinction paradigm, our current results and other recent findings have shown that this method is effective at reducing reinstatement and/or renewal of drug- (cocaine, morphine and heroin), sugarand fear-related memories in rodents and humans (Monfils et al, 2009; Clem and Huganir, 2010; Schiller et al, 2010; Flavell et al, 2011; Rao-Ruiz et al, 2011; Xue et al, 2012). Thus, in light of these new findings, post-retrieval extinction has the potential to be a clinically applicable therapy for the treatment of addiction and other psychiatric disorders. 


\section{Limitations of Post-Retrieval Extinction}

Although reconsolidation-based therapies offer a pragmatic approach to attenuate drug-related memories, subtle boundary conditions may limit the application of these treatments (Nader and Einarsson, 2010; Auber et al, 2013). For example, the strength, age and type of drug memory determine whether it is susceptible to reconsolidation-based amnestics. Additionally, the type and length of retrieval and extinction may also influence reconsolidation processes (Pedreira and Maldonado, 2003). In the current experiments, we show that a single post-retrieval extinction session is capable of reducing cocaine-primed reinstatement of $\mathrm{CPP}$ and relapse of CPP following protracted abstinence (drug-free and cocaine-primed), whereas other reports used multiple retrieval-extinction sessions to attenuate drug-related memories (Ma et al, 2012; Xue et al, 2012; Millan et al, 2013). Because experiences that produce new learning are required to initiate reconsolidation following reactivation (Pedreira et al, 2004; Morris et al, 2006; Sevenster et al, 2012, 2013), it is unclear whether multiple retrieval-extinction sessions are necessary, as activation of reconsolidation processes would likely diminish following repeated daily sessions. The behavioral paradigm used, however, may determine whether multiple retrieval-extinction sessions are needed to attenuate drug-related memories. Preliminary unpublished findings from our laboratory indicate that a single post-retrieval extinction session was not sufficient to reduce renewal of cocaine seeking in a self-administration paradigm (number of lever presses during renewal test: No Ret $=53 \pm 12$, Ret $=49 \pm 10 ; n=2$ and 3 for No Ret and Ret groups, respectively), whereas Xue et al (2012) showed that repeated post-retrieval extinction sessions attenuated renewal of cocaine and heroin self-administration. Lastly, some studies have shown that post-retrieval extinction was not effective at reducing fear or reward-related memories (Perez-Cuesta and Maldonado, 2009; Chan et al, 2010; Costanzi et al, 2011; Flavell et al, 2011; Ishii et al, 2012; Ma et al, 2012; Millan et al, 2013), although specific methodological and boundary conditions (species differences; behavioral paradigm used; type of reinforcer; length of conditioning, retrieval and/or extinction; time between retrieval and extinction training) may explain the divergent results between studies.

Ma et al (2012) recently reported that post-retrieval extinction does not completely erase morphine-related memories, as morphine CPP was reinstated by a priming injection following 4 weeks (but not at 1 week) of abstinence. However, we found that a cocaine prime following 30 days of abstinence did not reinstate cocaine CPP in animals that had retrieval training. This supports the possibility that our retrieval manipulation interfered with the memory of the cocaine-context association in a long-lasting fashion. Taken together, there appear to be certain conditions under which post-retrieval extinction training attenuates reward-related memories. However, further elucidation of the boundary conditions and molecular mechanisms by which post-retrieval extinction (and possibly the reversal, extinction-retrieval manipulation) (Millan et al, 2013) reduces drug-seeking behaviors is needed.

A few recent studies have started to shed some light on molecular targets involved in post-retrieval extinction. RaoRuiz et al (2011) showed that endocytosis of GluA2-AMPARs in the dorsal hippocampus is necessary for attenuation of contextual fear-related memories using the post-retrieval extinction protocol. Because GluA2-AMPARs also has a role in cocaine-evoked neuroplasticity and seeking behaviors (Boudreau et al, 2007; Mameli et al, 2007; Famous et al, 2008), it is possible that similar mechanisms regulate drug seeking during retrieval-extinction manipulations. In another study, Xue et al (2012) revealed that $\mathrm{PKM \zeta}$, an atypical constitutively active isoform of protein kinase $\mathrm{C}$, was elevated in the infralimbic cortex and decreased in the basolateral amygdala following post-retrieval extinction of cocaineseeking behaviors, though it is unclear if $\mathrm{PKM} \zeta$ has an essential role in post-retrieval extinction. Thus, these initial studies have revealed two potential molecular targets involved in post-retrieval extinction. However, additional studies are needed to generate a better understanding of the boundary conditions that govern memory reconsolidation, and further define the underlying molecular mechanisms involved in post-retrieval extinction and identify new reconsolidationbased targets for the treatment of drug addiction.

In summary, maladaptive learning and memory processes contribute importantly to the pathophysiology of several psychiatric disorders. Although cognitive-behavioral therapies have had limited success in the treatment of addiction and anxiety, recent studies suggest that post-retrieval extinction therapy has the potential to permanently attenuate drug- and fear-associated memories. Here, we showed that post-retrieval extinction blocks expression of cocaine memories following protracted abstinence, and that post-retrieval conditioning can be used to enhance rewardrelated memories. Moreover, our results revealed that a single post-retrieval extinction session may be effective in attenuating drug seeking under the proper conditions. These new data carry important implications for the treatment of addiction, as post-retrieval extinction might be used to diminish drug-context associations, and postretrieval reconditioning could be utilized to compete with drug rewards by strengthening and/or facilitating the restoration of natural reward processes in addicts. However, as most of the studies to date have used animals with limited drug histories, more work is needed to determine the efficacy of post-retrieval extinction in patients and/or animal suffering from chronic drug use. Future studies that address these unanswered questions will determine the clinical utility of post-retrieval extinction therapy to persistently reduce relapse behaviors.

\section{FUNDING AND DISCLOSURE}

The authors declare that they have no competing financial interests in relation to the work described. The authors declare that over the past 3 years, GA-J has received compensation from Ironwood Pharmaceuticals, Elsevier S.V., and the National Institutes of Health. However, no compensation was received for compounds or devices used in this research. GCS declares that he received no compensation from outside entities over the past 3 years.

\section{ACKNOWLEDGEMENTS}

We thank Jennifer Osborne and Michael E Smith for their excellent technical assistance. This work was supported by 
National Institute of Drug Abuse grants R01DA017289, R37DA06214, T32 DA007288, and C06 RR015455.

\section{REFERENCES}

Auber A, Tedesco V, Jones CE, Monfils MH, Chiamulera C (2013). Post-retrieval extinction as reconsolidation interference: methodological issues or boundary conditions? Psychopharmacology (Berl) 226: 631-647.

Barr AM, Fiorino DF, Phillips AG (1999). Effects of withdrawal from an escalating dose schedule of d-amphetamine on sexual behavior in the male rat. Pharmacol Biochem Behav 64: 597-604.

Boudreau AC, Reimers JM, Milovanovic M, Wolf ME (2007). Cell surface AMPA receptors in the rat nucleus accumbens increase during cocaine withdrawal but internalize after cocaine challenge in association with altered activation of mitogen-activated protein kinases. J Neurosci 27: 10621-10635.

Chan WY, Leung HT, Westbrook RF, McNally GP (2010). Effects of recent exposure to a conditioned stimulus on extinction of Pavlovian fear conditioning. Learn Mem 17: 512-521.

Clem RL, Huganir RL (2010). Calcium-permeable AMPA receptor dynamics mediate fear memory erasure. Science 330: 1108-1112.

Conklin CA, Tiffany ST (2002). Applying extinction research and theory to cue-exposure addiction treatments. Addiction 97: 155-167.

Costanzi M, Cannas S, Saraulli D, Rossi-Arnaud C, Cestari V (2011). Extinction after retrieval: effects on the associative and nonassociative components of remote contextual fear memory. Learn Mem 18: 508-518.

Crombag HS, Shaham Y (2002). Renewal of drug seeking by contextual cues after prolonged extinction in rats. Behav Neurosci 116: 169-173.

Famous KR, Kumaresan V, Sadri-Vakili G, Schmidt HD, Mierke $\mathrm{DF}, \mathrm{Cha} \mathrm{JH}$ et al (2008). Phosphorylation-dependent trafficking of GluR2-containing AMPA receptors in the nucleus accumbens plays a critical role in the reinstatement of cocaine seeking. J Neurosci 28: 11061-11070.

Flavell CR, Barber DJ, Lee JL (2011). Behavioural memory reconsolidation of food and fear memories. Nat Commun 2: 504.

Fricks-Gleason AN, Marshall JF (2008). Post-retrieval betaadrenergic receptor blockade: effects on extinction and reconsolidation of cocaine-cue memories. Learn Mem 15: 643-648.

Fuchs RA, Bell GH, Ramirez DR, Eaddy JL, Su ZI (2009). Basolateral amygdala involvement in memory reconsolidation processes that facilitate drug context-induced cocaine seeking. Eur J Neurosci 30: 889-900.

Gawin FH, Kleber HD (1986). Abstinence symptomatology and psychiatric diagnosis in cocaine abusers. Clinical observations. Arch Gen Psychiatry 43: 107-113.

Glickman SE (1961). Perseverative neural processes and consolidation of the memory trace. Psychol Bull 58: 218-233.

Harris GC, Aston-Jones G (2003a). Altered motivation and learning following opiate withdrawal: evidence for prolonged dysregulation of reward processing. Neuropsychopharmacology 28: 865-871.

Harris GC, Aston-Jones G (2003b). Critical role for ventral tegmental glutamate in preference for a cocaine-conditioned environment. Neuropsychopharmacology 28: 73-76.

Harris GC, Wimmer M, Aston-Jones G (2005). A role for lateral hypothalamic orexin neurons in reward seeking. Nature 437: 556-559.

Harris GC, Wimmer M, Byrne R, Aston-Jones G (2004). Glutamateassociated plasticity in the ventral tegmental area is necessary for conditioning environmental stimuli with morphine. Neuroscience 129: 841-847.
Hutton-Bedbrook K, McNally GP (2013). The promises and pitfalls of retrieval-extinction procedures in preventing relapse to drug seeking. Front Psychiatry 4: 14.

Hyman SE (2005). Addiction: a disease of learning and memory. Am J Psychiatry 162: 1414-1422.

Ishii D, Matsuzawa D, Matsuda S, Tomizawa H, Sutoh C, Shimizu E (2012). No erasure effect of retrieval-extinction trial on fear memory in the hippocampus-independent and dependent paradigms. Neurosci Lett 523: 76-81.

Lee JL, Milton AL, Everitt BJ (2006). Cue-induced cocaine seeking and relapse are reduced by disruption of drug memory reconsolidation. J Neurosci 26: 5881-5887.

Lee JL, Di Ciano P, Thomas KL, Everitt BJ (2005). Disrupting reconsolidation of drug memories reduces cocaine-seeking behavior. Neuron 47: 795-801.

Ma X, Zhang JJ, Yu LC (2012). Post-retrieval extinction training enhances or hinders the extinction of morphine-induced conditioned place preference in rats dependent on the retrieval-extinction interval. Psychopharmacology (Berl) 221: 19-26.

Mameli M, Balland B, Lujan R, Luscher C (2007). Rapid synthesis and synaptic insertion of GluR2 for mGluR-LTD in the ventral tegmental area. Science 317: 530-533.

McGaugh JL (1966). Time-dependent processes in memory storage. Science 153: 1351-1358.

Millan EZ, Milligan-Saville J, McNally GP (2013). Memory retrieval, extinction, and reinstatement of alcohol seeking. Neurobiol Learn Mem 101: 26-32.

Milton AL, Lee JL, Butler VJ, Gardner R, Everitt BJ (2008). Intraamygdala and systemic antagonism of NMDA receptors prevents the reconsolidation of drug-associated memory and impairs subsequently both novel and previously acquired drug-seeking behaviors. J Neurosci 28: 8230-8237.

Monfils MH, Cowansage KK, Klann E, LeDoux JE (2009). Extinction-reconsolidation boundaries: key to persistent attenuation of fear memories. Science 324: 951-955.

Morris RG, Inglis J, Ainge JA, Olverman HJ, Tulloch J, Dudai Y et al (2006). Memory reconsolidation: sensitivity of spatial memory to inhibition of protein synthesis in dorsal hippocampus during encoding and retrieval. Neuron 50: 479-489.

Nader K, Einarsson EO (2010). Memory reconsolidation: an update. Ann N Y Acad Sci 1191: 27-41.

Nader K, Schafe GE, Le Doux JE (2000). Fear memories require protein synthesis in the amygdala for reconsolidation after retrieval. Nature 406: 722-726.

O’Brien CP, Childress AR, McLellan T, Ehrman R (1990). Integrating systemic cue exposure with standard treatment in recovering drug dependent patients. Addict Behav 15: 355-365.

Pedreira ME, Maldonado H (2003). Protein synthesis subserves reconsolidation or extinction depending on reminder duration. Neuron 38: 863-869.

Pedreira ME, Perez-Cuesta LM, Maldonado H (2004). Mismatch between what is expected and what actually occurs triggers memory reconsolidation or extinction. Learn Mem 11: 579-585.

Perez-Cuesta LM, Maldonado H (2009). Memory reconsolidation and extinction in the crab: mutual exclusion or coexistence? Learn Mem 16: 714-721.

Rao-Ruiz P, Rotaru DC, van der Loo RJ, Mansvelder HD, Stiedl O, Smit $\mathrm{AB}$ et al (2011). Retrieval-specific endocytosis of GluA2AMPARs underlies adaptive reconsolidation of contextual fear. Nat Neurosci 14: 1302-1308.

Sanchez H, Quinn JJ, Torregrossa MM, Taylor JR (2010). Reconsolidation of a cocaine-associated stimulus requires amygdalar protein kinase A. J Neurosci 30: 4401-4407.

Sartor GC, Aston-Jones GS (2012a). A septal-hypothalamic pathway drives orexin neurons, which is necessary for conditioned cocaine preference. J Neurosci 32: 4623-4631. 
Sartor GC, Aston-Jones G (2012b). A septal-hypothalamic pathway drives orexin neurons, which is necessary for conditioned place preference. J Neurosci 32: 4623-4631.

Schiller D, Monfils MH, Raio CM, Johnson DC, Ledoux JE, Phelps EA (2010). Preventing the return of fear in humans using reconsolidation update mechanisms. Nature 463: 49-53.

Sevenster D, Beckers T, Kindt M (2012). Retrieval per se is not sufficient to trigger reconsolidation of human fear memory. Neurobiol Learn Mem 97: 338-345.

Sevenster D, Beckers T, Kindt M (2013). Prediction error governs pharmacologically induced amnesia for learned fear. Science 339: 830-833.

Shaham Y, Shalev U, Lu L, De Wit H, Stewart J (2003). The reinstatement model of drug relapse: history, methodology and major findings. Psychopharmacology (Berl) 168: 3-20.
Torregrossa MM, Corlett PR, Taylor JR (2011). Aberrant learning and memory in addiction. Neurobiol Learn Mem 96: 609-623.

Valjent E, Corbille AG, Bertran-Gonzalez J, Herve D, Girault JA (2006). Inhibition of ERK pathway or protein synthesis during reexposure to drugs of abuse erases previously learned place preference. Proc Natl Acad Sci USA 103: 2932-2937.

Volkow ND, Wang GJ, Telang F, Fowler JS, Logan J, Childress AR et al (2006). Cocaine cues and dopamine in dorsal striatum: mechanism of craving in cocaine addiction. J Neurosci 26: 6583-6588.

Xue YX, Luo YX, Wu P, Shi HS, Xue LF, Chen C et al (2012). A memory retrieval-extinction procedure to prevent drug craving and relapse. Science 336: 241-245. 